\title{
Online Recommender System for Personalized Nutrition Advice
}

\author{
Rodrigo Zenun Franco \\ Computer Science \\ University of Reading \\ Reading, United Kingdom \\ rodrigo.zenun.franco@pgr.reading.ac.uk
}

\begin{abstract}
The general recommendations for addressing noncommunicable diseases, which are responsible for two thirds of deaths globally, are mainly related to lifestyle changes, such as diet and physical activity. Challenges with encouraging healthy diets include gathering accurate information about dietary intake and delivering interventions that can influence behavior. Internet technologies offer excellent potential for addressing these challenges. Furthermore, personalized recommendations are more effective than general population-based recommendations at modifying health-related behavior in nutrition interventions.

The overall aim of this project is to design, develop and evaluate a recommender system able to assess dietary intake, using a validated Food Frequency Questionnaire (FFQ), and propose valid personalized nutrition advice for adults. It is investigating an effective way of providing personalized online dietary recommendations to increase diet quality at populationlevel and of considering an individual user's preferences, population data and experts' knowledge in the recommendation. The system is envisaged to be a web-based service, built with commercially available technologies, scalable, replicable, inexpensive and independent of any bespoke device (e.g. proprietary activity trackers).

Different levels of personalized advice will be evaluated via an online Randomised Control Trial (RCT) and surveys with nutrition professionals will be used for rating the advice proposed by the recommender system.
\end{abstract}

\section{KEYWORDS}

Nutrition; Diet; Food; Health; Personalization; Recommender systems; Behavior change

\section{INTRODUCTION}

Non-communicable diseases such as diabetes and cardiovascular diseases account for almost two thirds of deaths globally. The general recommendations for addressing these

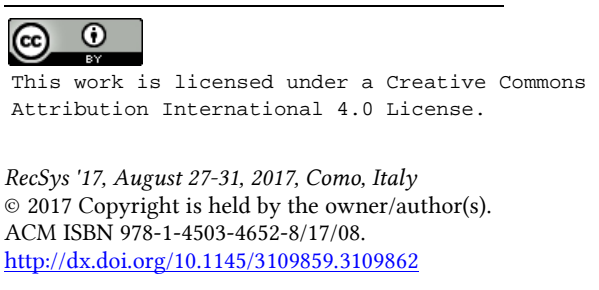

epidemics are related to lifestyle changes, mainly encouraging healthy diets, physical activity (PA) and the reduction of tobacco use and alcohol consumption [24]. The majority of face-to-face nutrition consultations in public services are only available to people with diagnosed conditions such as diabetes and obesity. There is a need for preventative initiatives for the general population, which are currently focused on public guidelines only.

A nutrition consultation cycle can be simplified as comprising three main steps: Assessment, Decision-making and provision of Advice. Typically, a person's dietary intake is assessed and then used as an input for decision-making, in order to provide feedback to the person. In developing an online system for personalized nutrition, all three steps are important to address.

Regarding the assessment stage, a valid method for online dietary intake recording is key. An important and longestablished retrospective nutrition assessment method is the Food Frequency Questionnaire (FFQ). It has been used in epidemiological studies for decades [22]. With this method, participants are asked to indicate frequencies and portion sizes for foods that they have consumed during a period of time (e.g. over the last month). FFQs have some accuracy limitations (e.g. calorie estimation), but, relative to some other methods such as 4-day food diaries, are more appropriate for initial dietary assessments aiming at personalized nutrition; FFQs are able to capture habitual dietary intake patterns, can be completed in a relatively short time by participants, and lend themselves to automated data processing []ㅡ.

After a person has completed an FFQ, researchers and nutrition professionals typically use guidelines to evaluate the diet quality of an individual. They may calculate the energy and nutrient content of the person's diet and compare this with current nutritional guidelines, in order to guide decision-making. However, food composition tables can contain more than a hundred nutrients and analysis of a person's nutrient profile can be complex and difficult to summarize into useful dietary advice. To address this challenge, a number of indexes of diet quality have been developed over the last decades (see [4] for a comprehensive report containing existing indexes of diet quality, published in 2005). Some of the indexes focus on local guidelines and others on specific target groups or diseases. One particular index of interest for this project is the United States Department of Agriculture (USDA)'s Healthy Eating Index (HEI), whose most recent version was published in 2010 [11]. In some cases, specific indexes have been created to correlate with particular diseases, such as the Alternative Healthy Eating Index (AHEI) which claims to predict the risk of chronic diseases [5], which is the 
main nutritional issue to be targeted by the system proposed in this project.

\section{RELATED WORK}

\subsection{Personalized nutrition}

Recent work in personalized nutrition intervention studies have shown that personalized recommendations based on diet are more effective than general population-based recommendations at modifying health-related behavior change [4]. In the Food4Me project, the advice was based on a decision tree, executed manually (i.e. by humans) during the study, and subsequently automated [7]. Details of the decision tree have not been published.

There are also studies investigating the personalization of recipes [10] and nutrition decisions [21], aiming to increase the acceptability of recommendations to the user.

\subsection{Diet and meal planning}

Experts Systems have been used in the nutrition field, mainly for meal planning [3][12]. Some systems applied Case-Based Reasoning (CBR) to propose a nutrient balanced meal plan [20]. Diet modeling was recently approached using constraint optimization techniques [19]. Besides meeting the nutritional guidelines, it is imperative to recommend recipes that individuals will like. Some systems proposed solutions based on recipes collected from the Internet [13]. The decision making process for nutrition advice is not discrete, opening an opportunity for addressing this challenge via fuzzy logic [16] .

\subsection{Dietary patterns in nutrition surveys}

Population surveys, such as the National Diet and Nutrition Survey (NDNS) in the UK [18], can provide insightful information for tailoring nutrition recommendations. The NDNS covers a representative sample of around 1,000 British residents per year. This type of survey offers potential for analyzing eating behaviors, using for example Topic Models [23] . Factor Analysis has potential to support the identification of food groups that correlate with each other and Cluster Analysis can identify clusters of individuals with similar dietary patterns [15].

\section{PROBLEM STATEMENTS}

The core challenge of this research is to design an online system that can automatically provide personalized nutrition advice that will be effective in changing people's diets. Besides factoring in an individual's dietary intake relative to general recommended dietary guidelines, it is important that the intervention takes into account personal information (e.g. phenotype, health conditions, dietary restrictions, etc.), population data (e.g. food availability and popularity) and users' preferences (e.g. preferred foods) in defining advice/feedback.

It is particularly important that this recommender system be able to consider participant's actual willingness to change their behavior. For instance, one could want to improve nutrition and yet be unwilling to decrease alcohol consumption, so the cost of the path for reducing the alcohol component would need to be recognized as higher than an alternative method for increasing the diet quality index, such as including more fruit for example. In other words, the system would need to take into account participants' interactions and propose different paths for increasing their diet quality.

Another key challenge in designing a nutrition recommendation system is the problem of how to train the system. In a typical recommender system, for example a medical diagnosis system, there is available data on the inputs (e.g. symptoms) and corresponding outputs (i.e. diagnosis/treatment). One of the main differences with a nutrition recommender system is that there is no existing openly-available dataset with people's case histories and the corresponding proposed diet modifications from nutrition professionals that could be used for training. Furthermore, differently from many common recommender systems (e.g. online shopping based on previous purchases), the food items that users like and consume the most are not necessarily the healthiest. In other words, the recommender system must consider factors other than users' preferences and prior consumption.

\section{RESEARCH QUESTIONS}

Considering the knowledge in this field obtained from other studies, this project proposes to answer a number of novel research questions:

- How to provide valid online personalized nutrition advice based only on individual's dietary intake and preferences (e.g. food likes and dislikes)?

- How to capture intelligence from nutrition experts and consider it in the recommender system?

- How to increase its level of personalization and improve its acceptability using historical data (e.g. other users of the system) and population data (e.g. national surveys)?

\section{METHODS}

My $\mathrm{PhD}$ research plan comprises three main stages, reflecting the questions presented in the previous section.

\subsection{Recommender system based on individual's dietary intake and preferences}

In my $\mathrm{PhD}$ research to date, I have designed and deployed an online personalized nutrition advice system called e-Nutri. It asks users to complete a FFQ (Fig. 1) which is based on one that has been previously validated [6], converts the dietary intake into nutrients, calculates the components of the AHEI (e.g. vegetables, fruits and sugar) [5] , derives a personalized nutrition recommendation based on the AHEI score, and presents the recommendation (Fig. 2), together with a progress report (Fig. 3). It also calculates the ideal weight range of the participants, based on the Body Mass Index (BMI), and provides feedback on their physical activity level, based on the Baecke questionnaire [1] .

The design of the user interface takes advantage of an existing design guideline (i.e. Google Material Design [17]) to present web components that are familiar to the participants. It is a fully responsive web application and uses professional cloud computing services.

In a deployment study, 163 participants positively evaluated a previous version of this system and the results are reported in [9]. Currently, the feedback is driven purely by the diet data, and the question of how best to balance nutritional healthiness against users' preferences in a way that maximizes the likelihood 


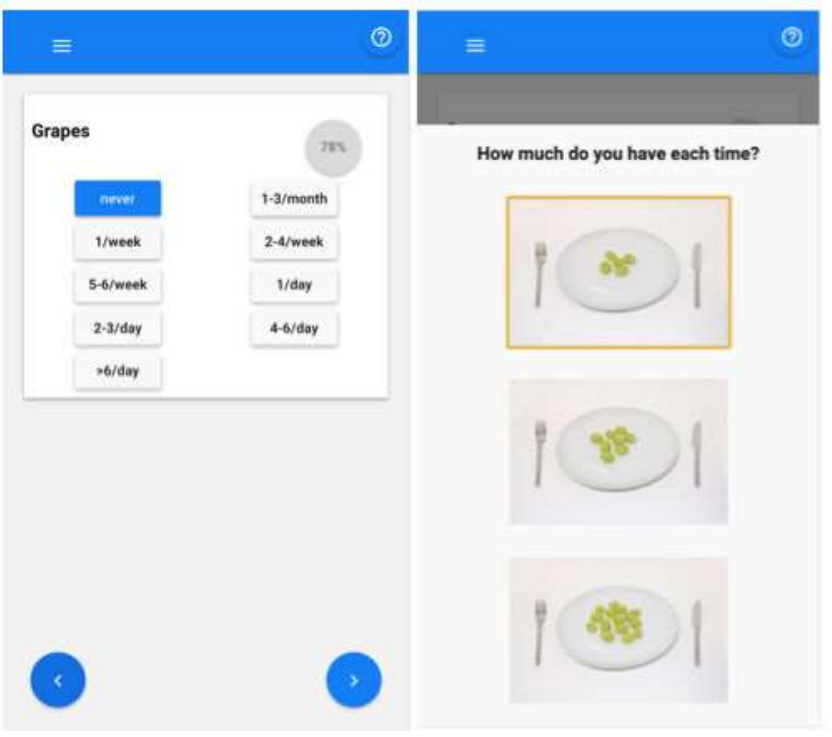

Figure 1: e-Nutri - Food frequencies and portion selections in smartphones

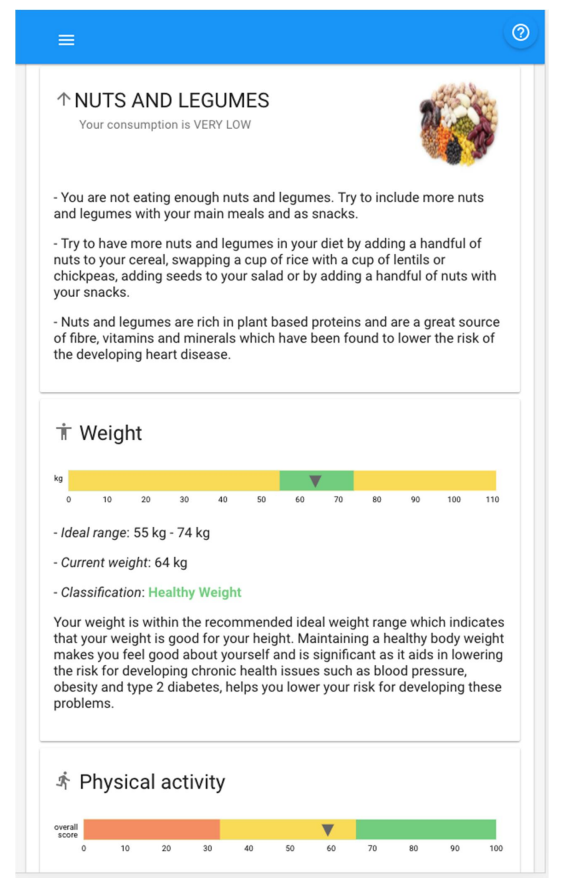

Figure 2: e-Nutri - Personalized report with nutrition advice, ideal weight range and physical activity level

of the user adopting healthier choices remains an open research challenge. A possible solution is to evaluate the quality of a diet using the AHEI, to identify multiple foods that can improve the AHEI score and to let the user choose from the multiple modifications. If the system also allows the users to rate the proposed modifications, that could act as further input to improve the recommendations. This type of approach seems to

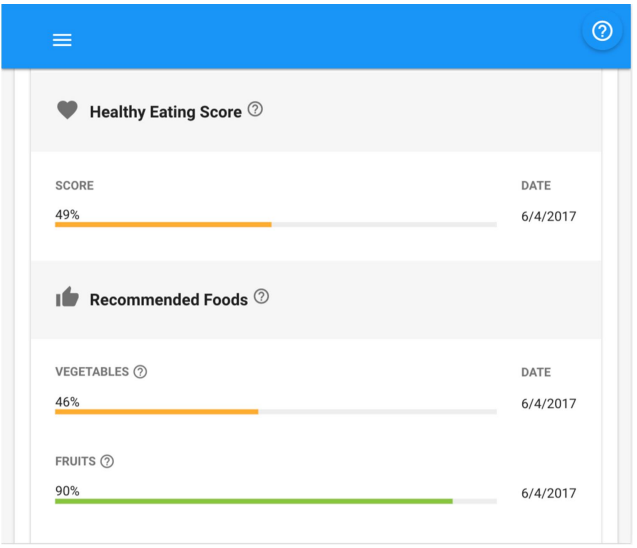

Figure 3: e-Nutri presenting the progress during the study

lend itself well to the use of node graphs, where the nodes represent the dietary intakes and vertices the possible changes [3]. Different from basic meal planning, it is important to have different weights (i.e. change acceptability) for the vertices, similar to map routing with different traffic conditions.

The system is also able to collect timestamps and device details directly from the web browser, and this data can offer insight about how people are using the system. After completing the FFQ, participants are requested to complete the System Usability Scale (SUS) [2] questionnaire for measuring acceptability of the nutrition assessment stage.

\subsection{Incorporating intelligence from nutrition experts}

Regarding incorporating intelligence from nutrition experts, the project also includes conducting a dietary feedback survey validating the advice provided by the e-Nutri software against professional nutrition standards. The dietary feedback survey will explore professional (Registered Dietitians and Nutritionists) agreement with the e-Nutri automated personalized nutrition advice. Participants $(n=32)$ will be recruited via professional associations (British Dietetic Association, UK Voluntary Register for Nutritionists) and invited to complete the survey online. The survey will present each participant with 2 scenarios, each comprising dietary intake analysis, individual characteristics and the designated advice. The scenarios $(n=16)$ will be drawn from a pool of FFQ responses (available to us from the Food4Me study [7]) based on four features (age, gender, BMI and HEI), and so have good ecological validity. The participants will be asked to audio record (via an online form) the most relevant nutrition recommendations to that hypothetical individual, using their professional judgment. After this stage, the participants will be allowed to access the nutrition advice generated by our recommender system and asked to rate their level of agreement, considering appropriateness, relevance and suitability, via 5-star style questions. Additional feedback will also be collected via audio or free text boxes. The results from this study will be used to refine and improve the recommendations provided by the system. 


\subsection{Incorporating intelligence from population data}

One alternative for addressing the lack of initial data to train the system is to capture intelligence from national nutrition surveys, which provide detailed dietary intake (e.g. food diaries) and socio-demographic (e.g. gender and age) data. It opens a possibility for detecting correlations, such as the most consumed fruits by a specific age range and gender, or for assessing which food substitutions are more likely to be acceptable.

Differently from FFQs, which use a limited food list, the nutrition surveys contain complete food diaries linked with extensive food composition tables (i.e. thousands of items). This type of dataset contains information that would not be captured even after intensive use of the system based only on the FFQ.

\subsection{Randomized Control Trial}

At the final stage of the project, the effectiveness of the nutrition recommendation will be evaluated, via an online RCT with the following groups ( $\mathrm{n}=100$ per group):

- Level 0 (Control group): web-based delivery of nonpersonalized dietary and physical activity exercise based on the UK general healthy eating guidelines.

- Level 1: Web-based delivery of personalized nutrition information and physical activity advice based on the individual's dietary intake, anthropometrics and physical activity levels.

- Level 2: As in Level 1, plus recommendations based on group/population preferences, as a way to improve the acceptability of the recommendation.

The effectiveness of the recommender system will be captured in terms of users' actual diet change, using the AHEI as an outcome measure. As well, the recommender system will be evaluated via questions regarding the users' perceived system effectiveness and quality [14] and the report design.

\section{CONCLUSIONS}

This project proposes to increase the acceptability, effectiveness and adoption of online nutrition services. This project will investigate an effective way of providing automated personalized online dietary recommendations in order to increase the diet quality of the population and ways considering user's preferences during the online recommendation.

This proposed system aims to increase effectiveness and acceptability using the following dimensions: dietary intake, user's preferences, other users' responses, population data and nutrition experts' knowledge.

To date, the $\mathrm{PhD}$ work has addressed a number of important challenges in usability of assessing dietary intake assessment, and proposing basic personalized diet recommendations based on dietary intake only. The remainder of the $\mathrm{PhD}$ research aims to design an intelligent system for personalized nutrition advice. Although the previous version of the system can be very useful and potentially contribute to healthier dietary habits, its decision engine is elementary computationally and deterministic (i.e. if two individuals have the same dietary intake information, they will receive the same advice, even if they have different preferences). In addition, the previous decision engine does not take into account important data sources, such as population data, historical data or individual's preferences. The hypothesis of the final study in the $\mathrm{PhD}$ is that these other data sources, combined with recommender system algorithms can increase both the acceptability and effectiveness of the nutrition advice.

\section{ACKNOWLEDGMENTS}

Thanks to Dr Faustina Hwang, Professor Julie A Lovegrove and Dr Rosalind Fallaize for the valuable support during my $\mathrm{PhD}$. Thanks to Keith Tam and Julia Castillo for the review of the report design. Thanks to the Food4Me (http://food4me.org) consortium for use of their portion size photographs. Rodrigo Zenun Franco is sponsored by $\mathrm{CNPq}$ (National Counsel of Technological and Scientific Development), from the Brazilian Government.

\section{REFERENCES}

[1] Baecke, J.A.J.A.H., Burema, J. and Frijters, J.E. 1982. A short questionaire for the measuremnet of habitual physical activity in epidemiological studies. The American journal of clinical nutrition. 36, November (Nov. 1982), 936-942.

[2] Brooke, J. 1996. SUS-a quick and dirty usability scale. 1996. Usability evaluation in industry. B.W. and A.M. PW Jordan, B. Thomas, ed. 4-7.

[3] Buisson, J.-C. 2008. Nutri-Educ, a nutrition software application for balancing meals, using fuzzy arithmetic and heuristic search algorithms. Artificial intelligence in medicine. 42, 3 (Mar. 2008), 213-27.

[4] Celis-Morales, C. et al. 2016. Effect of personalized nutrition on health-related behaviour change: evidence from the Food4me European randomized controlled trial. International fournal of Epidemiology. August (2016), dyw186.

[5] Chiuve, S.E., Fung, T.T., Rimm, E.B., Hu, F.B., McCullough, M.L., Wang, M., Stampfer, M.J. and Willett, W.C. 2012. Alternative dietary indices both strongly predict risk of chronic disease. Journal of Nutrition. 142, C (2012), 1009-1018.

[6] Fallaize, R, Forster, H. Macready, A.L. Walsh, M.C., Mathers, J.C., Brennan, L., Gibney, E.R., Gibney, M.J. and Lovegrove, J.A. 2014. Online dietary intake estimation: Reproducibility and validity of the Food4Me food frequency questionnaire against a 4-day weighed food record. Fournal of Medical Internet Research. 16, 8 (Aug. 2014), e190.

[7] Forster, H. et al. 2016. A dietary feedback system for the delivery of consistent personalized dietary advice in the web-based multicenter Food4Me study. Journal of Medical Internet Research. 18, 6 (Jun. 2016), e150.

[8] Forster, H., Fallaize, R., Gallagher, C., O’Donovan, C.B., Woolhead, C., Walsh, M.C., Macready, A.L., Lovegrove, J.A., Mathers, J.C., Gibney, M.J., Brennan, L. and Gibney, E.R. 2014. Online dietary intake estimation: the Food4Me food frequency questionnaire. Journal of medical Internet research. 16, 6 (Jan. 2014), e150.

[9] Franco, R.Z., Fallaize, R., Alwadhi, B., Lovegrove, J.A., Hwang, F., Alawadhi, B., Fallaize, R., Lovegrove, J.A. and Hwang, F. 2017. Design, Development and Usability Metrics of a Web-based Graphical Food Frequency Assessment System. FMIR Human Factors. 4, 2 (May 2017), e13.

[10] Freyne, J. and Berkovsky, S. 2010. Intelligent Food Planning: Personalized Recipe Recommendation. Proceedings of the 15th international conference on Intelligent user interfaces. (2010), 321-324.

[11] Guenther, P.M., Casavale, K.O., Reedy, J., Kirkpatrick, S.I., Hiza, H.A.B. Kuczynski, K.J., Kahle, L.L. and Krebs-Smith, S.M. 2013. Update of the Healthy Eating Index: HEI-2010. Fournal of the Academy of Nutrition and Dietetics. 113, 4 (Apr. 2013), 569-80.

[12] Harvey, M. and Elsweiler, D. 2015. Automated Recommendation of Healthy , Personalised Meal Plans. RecSys 2015: Proceedings of the 9th ACM conference on Recommender systems. (2015), 327-328.

[13] Harvey, M., Ludwig, B. and Elsweiler, D. 2013. You Are What You Eat: Learning User Tastes for Rating Prediction. Springer, Cham. 153-164.

[14] Knijnenburg, B.P. and Willemsen, M.C. 2015. Evaluating recommender systems with user experiments. Recommender Systems Handbook, Second Edition. (2015), 309-352.

[15] Krebs-Smith, S.M., Subar, A.F. and Reedy, J. 2015. Examining dietary patterns in relation to chronic disease: Matching measures and methods to questions of interest. Circulation. 132, 9 (2015), 790-793.

[16] Lee, C.-S., Wang, M.-H. and Lan, S.-T. 2014. Adaptive Personalized Diet Linguistic Recommendation Mechanism Based on Type-2 Fuzzy Sets and Genetic Fuzzy Markup Language. IEEE Transactions on Fuzzy Systems. 23, 5 (Oct. 2014), 1777-1802.

[17] Material design - Material design guidelines: 2016. https://material.google.com/ Accessed: 2016-10-26. 
[18] National Diet and Nutrition Survey: 2016 https://www.gov.uk/government/collections/national-diet-and-nutrition-survey. Accessed: 2017-06-28.

[19] Probst, Y., Morrison, E., Sullivan, E. and Dam, H.K. 2016. First-Stage Development and Validation of a Web-Based Automated Dietary Modeling Tool: Using Constraint Optimization Techniques to Streamline Food Group and Macronutrient Focused Dietary Prescriptions for Clinical Trials. Fournal of Medical Internet Research. 18, 7 (Jul. 2016), e190.

[20] Ravana, S.D., Rahman, S.A. and Chan, H.Y. 2006. Web-Based Diet Information System with Case-Based Reasoning Capabilities. International fournal of Web Information Systems. 2, 3/4 (2006), 154-163.
[21] Schäfer, H. 2016. Personalized Support for Healthy Nutrition Decisions. Proceedings of the 10th ACM Conference on Recommender Systems (2016).

[22] Welch, A.A., Luben, R., Khaw, K.T. and Bingham, S.A. 2005. The CAFE computer program for nutritional analysis of the EPIC-Norfolk food frequency questionnaire and identification of extreme nutrient values. Journal of Human Nutrition and Dietetics. 18, 2 (2005), 99-116.

[23] White, R., Harwin, W.S., Holderbaum, W. and Johnson, L. 2015. Investigating Eating Behaviours Using Topic Models. 2015 IEEE 14th International Conference on Machine Learning and Applications (ICMLA) (Dec. 2015), 265-270.

[24] WHO 2010. Global status report on noncommunicable diseases 2010. 